\title{
Salivary alpha amylase activity in pregnant and non- Pregnant females
}

\author{
Jyotsna Bhagirath Jaju ${ }^{1 *}$, PraveenaVithpala ${ }^{2}$, Amit Ashokkumar Bharadiya ${ }^{3}$, Bhavani Doddi ${ }^{4}$, Sravya Poduri $^{5}$ \\ ${ }^{1,5}$ Senior Resident, ${ }^{2}$ Assistant professor, ${ }^{3}$ Consultant, ${ }^{4}$ MBBS Student, ${ }^{\mathbf{1 , 2 5}}$ Dept. of Biochemistry, ${ }^{\mathbf{1 , 5}}$ All India Institute of Medical Sciences, \\ Jodhpur, Rajasthan, ${ }^{2}$ Employees State Insurance Corporation Medical College, Hyderabad, Telangana, ${ }^{4}$ Medipulse Hospital, Jodhpur, \\ Rajasthan, India
}

\section{Corresponding Author: Jyotsna Bhagirath Jaju}

Email: Jo_26387@yahoo.co.in

Received: $7^{\text {th }}$ February, 2019

Accepted: $6^{\text {th }}$ April, 2019

\begin{abstract}
Introduction: Pregnancy demands various physiological changes to be adapted by the body for growing fetus. Stress of pregnancy gives rise to altered hormonal release from hypothalamic-pituitary adrenal (HPA) and sympatho-adrenal-medullary (SAM) axis. Present study aimed to estimate salivary amylase activity in pregnant and non- pregnant females.

Materials and Methods: In this cross sectional study we have selected 65 pregnant females and compared with 22 non- pregnant females. Pregnant females were grouped into three according to trimesters. Non stimulated saliva sample was collected from study participants. Salivary amylase activity was measured by coupled enzymatic assay.

Results: Significant increase in salivary amylase in pregnant as compared to non-pregnant females was observed $(p=0.019)$. There was significant difference in salivary amylase between groups of pregnant females $(\mathrm{p}<0.001)$. There was no statistically significant relation between salivary amylase and number of pregnancies $(p=0.08)$.

Conclusion: Increased salivary amylase in pregnancy can be due to increased physical and physiological stress in pregnancy. Salivary amylase was studied as a marker of stress. Stress leads to increased sympathetic activity which in turn leads to increased salivary amylase. Saliva collection is non- invasive technique, easy to perform, less skillful and can be done repeatedly so it can be easily used for monitoring increased sympathetic activity.
\end{abstract}

Keywords: Salivary alpha amylase, Pregnancy, Non- pregnant, Stress, Sympathetic nervous system.

\section{Introduction}

Pregnancy requires various physiological changes for the growth of fetus. It alters human chorionic gonadotropin (HCG), progesterone and estrogen hormone levels in body. These alterations are responsible for the cardiovascular, genitourinary, respiratory, hematological and endocrine changes during pregnancy. ${ }^{1}$ Pregnancy also leads to altered hormone release from hypothalamic pituitary adrenal (HPA) and sympatho-adrenal-medullary (SAM) axis. ${ }^{2}$

Alpha amylase is most abundant protein present in saliva. ${ }^{3}$ It is secreted from acinar cells of salivary glands by activation of beta 1 adrenoreceptors. ${ }^{4}$ It is calcium containing metalloenzyme. It hydrolyzes starch by breaking alpha 1-4 glycosidic linkage, into maltose, maltrotriose and larger oligosaccharides. ${ }^{5}$ Salivary alpha amylase (sAA) production is independent of saliva flow rate. ${ }^{6}$ Parotid, submandibular and sublingual are major salivary glands which are innervated by sympathetic and parasympathetic nerves. Stimulation of these glands leads to production of salivary proteins. ${ }^{7}$ So, salivary alpha amylase can be the good candidate substance for measuring the autonomic activity. Human and animal studies showed marked elevation of sAA activity due to different physical and psychological stress. ${ }^{2}$ As pregnancy is a stressful condition in female's life, present study aimed to observe sAA activity in pregnant females and compare with non-pregnant. We also aimed to study sAA activity in different trimester of pregnancy to observe trend of sAA in pregnancy.

Saliva as a diagnostic fluid, has gained popularity over past few decades. Salivary biochemical parameters like cortisol have been used to assess HPA axis. ${ }^{8}$ Collection of saliva is non-invasive and painless procedure. It is also easy and economical. Various biochemical parameters like glucose, urea, creatinine and cholesterol measurement in saliva are under study. ${ }^{9}$ In future saliva can be used as a diagnostic fluid for different disease diagnosis and monitoring.

\section{Materials and Methods}

This was a cross-sectional study, approved by Institutional Ethical committee. Study participants were pregnant females attending antenatal outpatient department at our Institute. In this study pregnancy was confirmed by positive urine pregnancy test or ultrasonography report showing the live intrauterine pregnancy. Pregnant females having the history of diabetes mellitus, hypertension, psychiatric illness, dental problem and any other chronic disease were excluded from study. Females in follicular phase of menstrual cycle considered as non-pregnant group. These females were non-diabetic, non-hypertensive, not using oral contraceptives and not having any psychiatric illness. Informed written consent was obtained from all study participants.

Pregnant females $(n=65)$ of different trimesters were compared with non-pregnant females $(n=22)$. Pregnant were grouped into three according to trimester of pregnancy. Group I had 25 females of first trimester, group II had 19 females of second trimester and group III had 21 females of third trimester. Non- pregnant females were in follicular phase of menstrual cycle. Study participants were instructed 
before sample collection regarding, not to eat one hour before sample collection and rinse mouth before saliva collection. Non stimulated whole saliva sample was collected by spitting method. Saliva was stored at $-4^{\circ} \mathrm{C}$. Saliva was diluted 1:250 times before analysis. Salivary amylase was estimated by using ethylidene-G7-PNP as a substrate. Amylase activity is directly proportional to amount of paranitrophenol liberated, which was measured at $410 \mathrm{~nm}^{.10}$

Sample size was calculated using open Epi software. Mean and standard deviation (SD) of sAA activity in pregnant and non-pregnant were used; with $0.05 \%$ alpha error and $80 \%$ power. ${ }^{11}$ Statistical analysis was done in graph pad prism software and Microsoft excel. $P$ value $<0.05$ was considered as significant. All values were expressed as mean \pm SD. Salivary amylase levels of pregnant and non- pregnant women were compared by student $t$ test. The sAA activities in between the groups were compared by one way ANOVA.

\section{Results}

Table 1 shows baseline characteristics of all study participants. Mean age among the pregnant females $(n=65)$ was $25.67 \pm 2.87$ years and $28.9 \pm 5$ years among nonpregnant females $(n=22)$. There was no statistically significant difference between systolic and diastolic blood pressure. The mean hemoglobin concentration among pregnant $11.7 \pm 1.3 \mathrm{gm} / \mathrm{dl}$ was more than the non-pregnant $10.9 \pm 2.1 \mathrm{gm} / \mathrm{dl}$ females, which was statistically significant $(\mathrm{p}=0.01)$

There was significant increase in level of SAA activity in pregnant females $108.14 \times 10^{3} \pm 71.74 \times 10^{3} \mathrm{IU} / \mathrm{L}$ as compared to non- pregnant $70.6 \times 10^{3} \pm 30.68 \times 10^{3} \mathrm{IU} / \mathrm{L}$, $(\mathrm{p}$ $=0.019)$. We have observed difference in sAA activity in pregnant females of different trimester. First trimester had sAA activity $47.01 \times 10^{3} \pm 15.5 \times 10^{3} \mathrm{IU} / \mathrm{L}$, second trimester had sAA activity $98.4 \times 10^{3} \pm 38.3 \times 10^{3} \mathrm{IU} / \mathrm{L}$, third trimester had sAA activity $189.7 \times 10^{3} \pm 55.9 \times 10^{3}$ IU/L. Table 2 shows sAA activity in different trimesters. Third trimester women had statistically significant increase in sAA activity as compared to first and second trimester women, $(\mathrm{p}<0.001$. Fig. 1 is comparison of sAA activity in pregnant and nonpregnant women. The sAA activity in different trimester is shown in Fig. 2 which shows increased salivary amylase in III group as compare to II and I group.

Within 65 pregnant women 28 were primigravida, 17 were pregnant for $2^{\text {nd }}$ time, 15 were pregnant for $3^{\text {rd }}$ time, 4 were pregnant for $4^{\text {th }}$ time and single women pregnant for $5^{\text {th }}$ time. There was no significant difference in the sAA levels and number of pregnancies $(\mathrm{p}=0.08)$ which was depicted in Table 3 .

Table 1: Baseline characteristics of study participants

\begin{tabular}{|l|c|c|c|}
\hline \multicolumn{1}{|c|}{ Parameter } & Non pregnant Mean \pm SD & Pregnant Mean \pm SD & P value \\
\hline Age (years) & $28.9 \pm 5$ & $25.67 \pm 2.87$ & 0.03 \\
\hline Blood pressure systolic $(\mathrm{mmHg})$ & $114.9 \pm 10.4$ & $115.9 \pm 13.3$ & 0.74 \\
\hline Blood pressure diastolic $(\mathrm{mmHg})$ & $71 \pm 14.9$ & $74.5 \pm 8.6$ & 0.18 \\
\hline Pulse rate $($ rate/min) & $71.9 \pm 4.3$ & $112.2 \pm 10$ & 0.01 \\
\hline Hemoglobin $(\mathrm{gms} / \mathrm{dl})$ & $10.9 \pm 2.1$ & $11.7 \pm 1.3$ & 0.01 \\
\hline Salivary amylase $\mathrm{x} 10^{3}(\mathrm{IU} / \mathrm{L})$ & $70.6 \pm 30.6$ & $108.1 \pm 71.7$ & 0.019 \\
\hline
\end{tabular}

Table 2: Salivary amylase activity in females of different trimesters

\begin{tabular}{|c|c|c|c|c|}
\hline Parameter & Group I & Group II & Group III & P value \\
\hline Salivary amylase $\mathrm{x} 10^{3}(\mathrm{IU} / \mathrm{L})$ & $47.01(15.5)$ & $98.4(38.3)$ & $189.7(55.9)$ & $<0.001$ \\
\hline
\end{tabular}

Table 3: Salivary amylase activity in females and number of times pregnancy

\begin{tabular}{|c|c|c|c|c|c|c|}
\hline Parameter & $\mathbf{1}^{\text {st }}(\mathbf{2 8})$ & $\mathbf{2}^{\text {nd }}(\mathbf{1 7})$ & $\mathbf{3}^{\text {rd }}(\mathbf{1 5})$ & $\mathbf{4}^{\text {th }}(\mathbf{4})$ & $\mathbf{5}^{\text {th }}(\mathbf{1})$ & P value \\
\hline Salivary amylase $x 10^{3}(\mathrm{IU} / \mathrm{L})$ & $85.5(55.2)$ & $140.4(76.6)$ & $111.8(83.1)$ & $100.1(49.0)$ & 168.0 & 0.08 \\
\hline
\end{tabular}

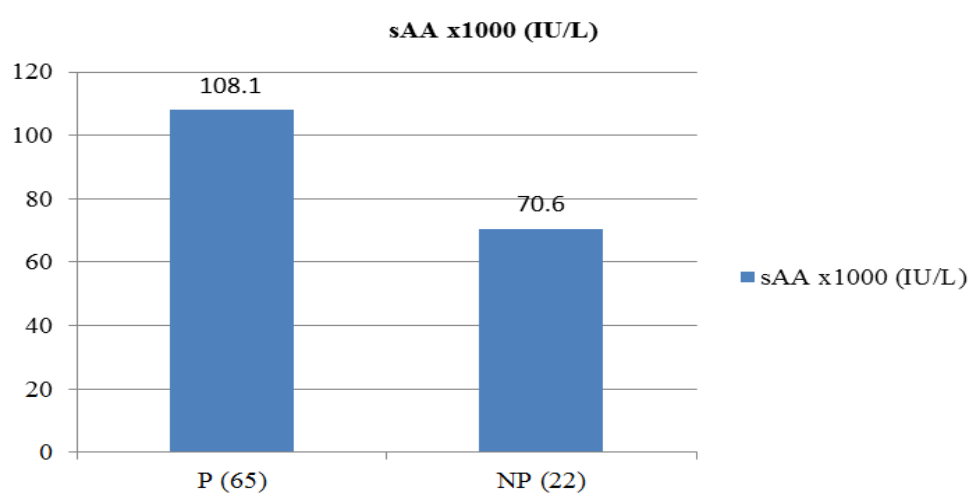

Fig. 1: Mean salivary amylase in pregnant and non-pregnant 


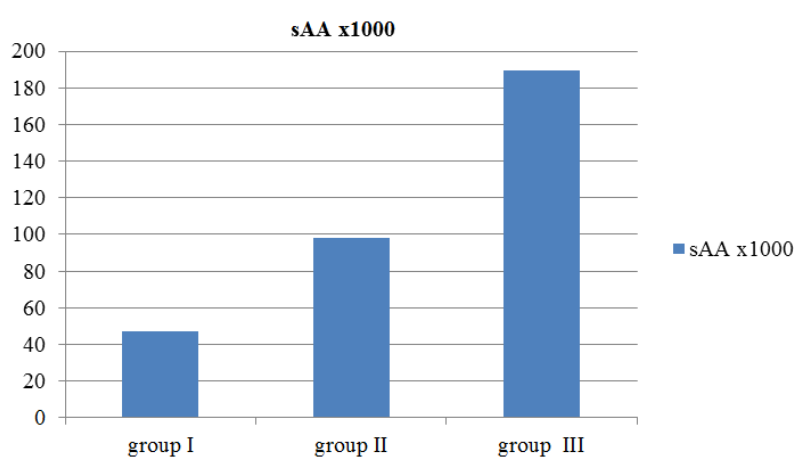

Fig. 2: Salivary alpha amylase activity in different trimester

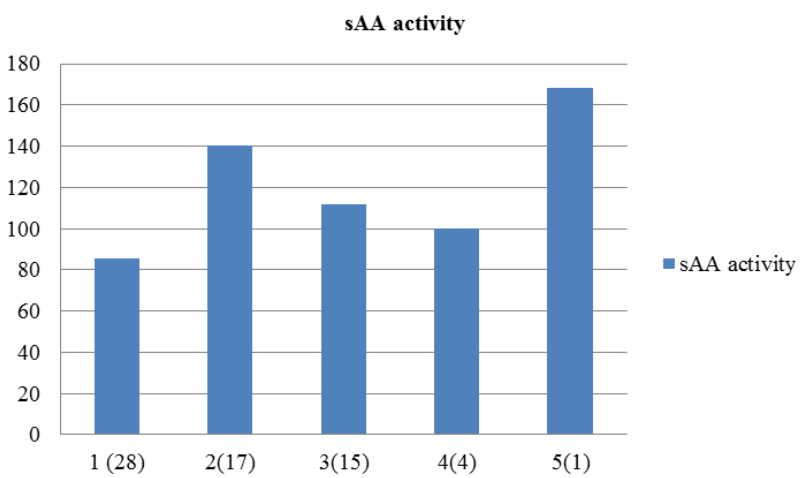

Fig. 3: Mean sAA activity and number of times pregnancy

\section{Discussion}

Primary aim of our study was to measure and compare the salivary amylase activity in pregnant and non- pregnant females. We observed statistically significant increase in sAA activity in pregnant women as compared to nonpregnant. We also observed sAA activity vary according to trimester of pregnancy. Third trimester of pregnancy has more increase in SAA activity as compare to second and first trimester. As per our knowledge present study is the first to show the changes in sAA activity and number of pregnancies and there was no significant difference in sAA activity and number of pregnancies.

Major salivary gland parotid is innervated by glossopharyngeal nerve and submandibular by facial nerve. parasympathetic nerve supply is from thoracic segment of spinal cord. ${ }^{12}$ Response to stress is determined by hypothalamic-pituitary -adrenal (HPA) axis and sympathetic adreno medullary (SAM) nervous system. HPA leads to the production of hormones which finally leads to release of cortisol from adrenal gland. Measurement of cortisol in serum or saliva indicates HPA axis. ${ }^{13}$ Sympathetic activation leads to increased heart rate, blood pressure, dilatation of pupil etc. Salivary alpha amylase can be indicator of sympathetic activity as salivary glands are innervated by sympathetic nervous system. ${ }^{12}$

The sAA present in highest concentration in saliva as it is produced locally. Salivary cortisol is secreted into saliva. Measuring sAA is easy and cheap as compare to salivary cortisol. Serum and salivary cortisol is studied in detail as a marker of different physical and psychological stress.
Salivary cortisol and salivary amylase had shown significant positive correlation in stressful conditions. So, measurement of sAA may have advantage over salivary cortisol. ${ }^{14}$

The relation between sAA and pregnancy is controversial. Salvolini E et al and Rio R et al. showed increase in sAA activity during the pregnancy. ${ }^{14-16}$ Cijeak M showed no relation between sAA activity and pregnant women of different gestational age. ${ }^{17}$ Giesbrecht F observed no relation between gestational age and maternal demographics with diurnal sAA. ${ }^{18}$ LaineM et al. observed no change in salivary amylase in pregnant and nonpregnant women. Studies where sAA activity studied with psychiatric conditions in pregnancy showed increased sAA activity in late pregnancy depression as compare to normal. Changes in the sympathetic nervous system causes vasoconstriction, which leads to decreased blood flow to placenta, affects the fetal outcome in pregnant females. ${ }^{19}$

Present study participants were not having mental stress. They were not on any antipsychotic medication or behavioral therapy. The sAA which was increased, can be due to increased sympathetic activity in pregnancy. ${ }^{20}$ There is no physiological reference range for salivary amylase for different age and sex groups, also special conditions like pregnancy.

Salivary amylase which is being used as a marker of stress in different psychological and physical stressful condition but to apply it for pregnant population requires special consideration. As pregnancy is stressful condition, it leads to increase in salivary amylase. As stress in pregnancy is not consistent salivary amylase increases as pregnancy increases. Pregnancy with other psychological stress will lead to more increase in salivary amylase. So prediction of increased salivary amylase in pregnant population is difficult.

In present study we have not observed statistically significant difference in sAA and number of pregnancies of female. The physiological change of pregnancy which leads to stress is almost same in each pregnancy can be the reason for that. In study pregnant females were not having bad obstetrics history. Out of 65 pregnant 28 were primigravida. Effect of bad obstetrics history and salivary amylase can be studied in future.

In present study we have not assessed salivary flow rate, as it does not interfere with sAA activity. ${ }^{21}$ Small sample size, stress was not assessed with existing stress scale are limitations of study. Further study with large sample size and continuous monitoring of salivary alpha amylase throughout pregnancy will provide clear idea.

\section{Conclusion}

Salivary amylase is increased in pregnancy. Levels vary in different trimester of pregnancy. There is no difference in salivary amylase and number of pregnancy. Salivary glands, which are innervated by sympathetic nerves, on stimulation increases amylase. Collection of saliva is non- invasive technique, easy to perform, less skillful and can be done repeatedly so it can be easily used for monitoring increased sympathetic activity. 


\section{Conflict of Interest: None.}

\section{References}

1. Graça L, Centeno M. Medicina Materno-Fetal. Lisboa. 2005:327-32.

2. De Weerth C, Buitelaar JK. Physiological stress reactivity in human pregnancy - a review. Neurosci Biobehavioral Rev 2005;29(2):295-312.

3. Zakowski JJ, Bruns DE. Biochemistry of human alpha amylase isoenzymes. CRC Crit Rev Clin Lab Sci 1985;21(4):283-322.

4. Schneyer CA, Hall HD. Effects of varying frequency of sympathetic stimulation on chloride and amylase levels of saliva elicited from rat parotid gland with electrical stimulation of both autonomic nerves. Proc Soc Exp Biol Med 1991;196(3):333-7.

5. Baum BJ. Principles of saliva secretion. Ann N York Acad Sci 1993;694(1):17-23.

6. Rohleder N, Wolf JM, Maldonado EF, Kirschbaum C. The psychosocial stress-induced increase in salivary alpha-amylase is independent of saliva flow rate. Psychophysiology 2006;43(6):645-52.

7. Garrett JR, Ekström J, Anderson LC. Neural mechanisms of salivary gland secretion: Karger Medical and Scientific Publishers; 1999.

8. Kirschbaum C, Hellhammer DH. Salivary cortisol in psychoneuroendocrine research: recent developments and applications. Psychoneuroendocrinology 1994;19(4):313-33.

9. Gupta A, Singh SK, Padmavathi B, Rajan S, Mamatha G, Kumar $\mathrm{S}$ et al. Evaluation of correlation of blood glucose and salivary glucose level in known diabetic patients. J Clin Diagn Res 2015;9(5):ZC106.

10. Lorentz K. International Federation of Clinical Chemistry (IFCC). Approved Recommendation on IFCC methods for the measurement of catalytic concentration of enzymes. Part 9. IFCC Method for $\alpha$-Amylase (1, 4- $\alpha$-D-Glucan 4Glucanohydrolase, EC 3.2. 1.1). Clin Chem Lab Med 1998;36:185-203.

11. Skoskiewicz-Malinowska K, Kaczmarek U. The Level of Salivary alpha-Amylase Activity During Pregnancy. Dent Med Problems 2013;50(1):30-7.
12. Bear MF, Connors BW, Paradiso MA. Neuroscience: Lippincott Williams \& Wilkins; 2007.

13. Chrousos GP. Stress and disorders of the stress system. Nat Rev Endocrinol 2009;5(7):374.

14. Abrão ALP, Leal SC, Falcão DP. Salivary and serum cortisol levels, salivary alpha-amylase and unstimulated whole saliva flow rate in pregnant and non-pregnant. Rev Bras Ginecologia Obstet 2014;36(2):72-8.

15. Salvolini E, Di Giorgio R, Curatola A, Mazzanti L, Fratto G. Biochemical modifications of human whole saliva induced by pregnancy. BJOG: Int J Obstet Gynaecol 1998;105(6):656-60.

16. Rio R, Azevedo Á, Simões-Silva L, Marinho J, Silva MJ, Sampaio-Maia B et al. The biochemistry of saliva throughout pregnancy. Med Express 2015;2(5).

17. Ciejak M, Olszewska M, Jakubowska K, Zebiełowicz D, Safranow K, Chlubek D et al, editors. Activity of alphaamylase and concentration of protein in saliva of pregnant women. Annales Academiae Medicae Stetinensis; 2007.

18. Giesbrecht GF, Granger DA, Campbell T, Kaplan B, Team AS. Salivary alpha-amylase during pregnancy: Diurnal course and associations with obstetric history, maternal demographics, and mood. Dev psychobiology 2013;55(2):156-67.

19. Braithwaite EC, Ramchandani PG, Lane TA, Murphy SE. Symptoms of prenatal depression are associated with raised salivary alpha-amylase levels. Psychoneuroendocrinology 2015;60:163-72.

20. Jarvis SS, Shibata S, Bivens TB, Okada Y, Casey BM, Levine BD et al. Sympathetic activation during early pregnancy in humans. J Physiol 2012;590(15):3535-43.

21. van Stegeren AH, Wolf OT, Kindt M. Salivary alpha amylase and cortisol responses to different stress tasks: impact of sex. Int J Psychophysiology 2008;69(1):33-40.

How to cite this article: Jaju JB, Vithpala $P$, Bharadiya AA, Doddi B, Poduri S, Salivary alpha amylase activity in pregnant and non- Pregnant females. Indian J Obstet Gynecol Res 2019;6(2):165-168. 